\title{
Phase field modeling of phase boundary motion due to transport-limited electrochemical reactions
}

\author{
A. Powell ${ }^{1} \&$ W. Pongsaksawad ${ }^{2}$ \\ ${ }^{1}$ Veryst Engineering LLC, Needham, Massachusetts, USA \\ ${ }^{2}$ National Metal and Materials Technology Center, Khlong Luang, \\ Pathumthani, Thailand
}

\begin{abstract}
A new phase field model is presented for simulation of phase boundary motion due to transport-limited electrochemical reactions. The model consists of CahnHilliard diffusion based on a statement of free energy with an electrostatic energy term, and conservation of charge. It is shown that under assumptions of negligible charge transfer resistance (mass transfer dominance) and rapid charge redistribution, the conservation of charge equation reduces to zero divergence of current density. When simulating electrolytic metal oxidation/reduction with an unsupported electrolyte, the model reproduces analytical models of cathode interface stability. It can also simulate electronically mediated reactions at separate interfaces, such as those occurring in metallothermic reduction processes. Results are presented for both unsupported and supported electrolytes, and both solidstate transformations and those involving fluid flow, including fluid-structure interactions using the Mixed Stress model for diffuse interface fluid-structure modeling.
\end{abstract}

Keywords: electrochemistry, mathematical modeling, phase field, fluid flow, stability analysis, dendrite, streamer, titanium, steelmaking.

\section{Introduction}

Electrolysis enjoys widespread use for extraction of metals from their ores or aqueous solutions. However, metal electrodeposition very often results in a rough surface or dendrites due to a Mullins-Sekerka instability at the cathode/electrolyte 
interface. Designing around this problem involves $e . g$. using additives in aqueous processes or limiting the current in high-temperature systems.

The few tools available to the designer for modeling the onset and development of such instabilities can be roughly categorized into: linear stability theory, particle models such as Monte Carlo simulations, and continuum models which track the interface such as finite element models with Lagrangian interface descriptions and level set methods.

Continuum models which explicitly track the interface such as that of Cao et al. [1] work sufficiently well under some circumstances but break down when the interface topology changes. The level set method [2] tracks interface propagation over time based on the curvature-dependent speed. This method has been used to model roughness evolution during superconformal electrodeposition with additives and catalysts in the electrolyte [3, 4]. Unfortunately, it requires that the interface dynamics be understood a priori, making it difficult to extend to complex systems with many components.

The phase field methodology derives its governing equations from a thermodynamic equation for free energy. Guyer et al. have developed a general formulation for phase field modeling of electrodeposition and electrodissolution [5]. Although their approach includes the detail of charge distribution at the interface, it is currently limited in two ways: it requires discretization of the electrochemical double layer, limiting its use to nanometer-scale systems, and it is a numerically challenging technique, such that it has to date only been solved in one dimension.

When limited by mass transfer, the effect of charge transfer resistance at the interfacial double layer is negligible, removing the need to discretize the double layer, and permitting simulation of larger systems on scales up to millimeters and beyond. The resulting phase field method, described by the authors in detail elsewhere [6], considerably simpler than that of Guyer, but limited to those situations where charge transfer resistance can be neglected. This paper outlines that method as applied to a liquid-liquid iron/iron oxide system, and a ternary titanium/magnesium/chlorine system without flow.

\section{Binary iron/iron oxide model}

Electric Field-Enhanced Smelting and Refining (EFESR) was invented by Uday Pal [7] to drive the reaction between $\mathrm{FeO}$ in steelmaking slag and carbon in hot metal. This process operates by two half-cell reactions at the cathode (above) and anode (hot metal-slag interface) respectively:

$$
\begin{aligned}
\mathrm{Fe}^{2+}+2 \mathrm{e}^{-} & \Rightarrow \mathrm{Fe} \\
\mathrm{C}+\mathrm{O}^{2-} & \Rightarrow \mathrm{CO}+2 \mathrm{e}^{-}
\end{aligned}
$$

This has several beneficial effects: it increases the yield of steelmaking (about 2\% of the iron is lost to the slag), reduces foaming, and removes carbon from hot metal, possibly reducing the need for subsequent vacuum degassing. For stainless 
steel, it also removes chromium oxide from the slag, bringing both economic and environmental benefits.

The kinetics of the overall process are limited by mass transfer of ferrous ions to the cathode. But the cathode reaction in equation 1 forms liquid iron, which grows as the liquid equivalent of dendrites, called streamers in the aluminum literature, which dart out into the fresh slag and enhance the mass transfer coefficient considerably. Streamers then break up into droplets due to the Rayleigh instability of a liquid tube; these droplets then sink down to join the liquid hot metal below.

Because the kinetics of the process are determined by the formation and breakup of streamers, a model which describes them would aid in the design of a solid cathode, flow conditions, magnetic fields, etc., to optimize mass transfer.

\subsection{Thermodynamics}

A phase field formulation for coupled fluid flow, diffusion, electromigration, and transport-limited electrochemical reactions was developed by Dussault [8], and expanded by Pongsaksawad and Powell [6]. This model begins with a dimensionless concentration variable $C$ which is zero in the $\mathrm{FeO}$ slag and one in the iron metal, and uses a very simplistic polynomial function for the homogeneous free energy with minima at zero and one:

$$
\Psi(C)=C^{2}(1-C)^{2} .
$$

Following the Cahn-Hilliard formulation [9], as extended by the Guyer electrochemistry model $[5,10]$, the total chemical free energy adds terms for the concentration gradient and electrical potential:

$$
F=\int\left(\beta \Psi(C)+\frac{\alpha}{2}|\nabla C|^{2}+F \Phi \sum z_{i} c_{i}\right) d V
$$

where $\Phi$ is the electrical potential, $F$ is the Faraday constant, and $z_{i}$ and $c_{i}$ are the charge and concentration (in moles/volume) of each species. Like the chemical potential in the Cahn-Hilliard model, the total electrochemical potential in the slag is the variational derivative of the free energy functional:

$$
\mu_{\text {slag }}=\frac{\delta F}{\delta C}=-\alpha \nabla^{2} C+\beta \Psi^{\prime}(C)+4 F \rho_{M} \Phi .
$$

Charge neutrality requires that the iron charge be given by:

$$
z_{\mathrm{Fe}}(C)=\frac{2(1-C)}{(1+C)} .
$$

Because iron electromigration flux is proportional to its charge $z_{F e}$, the electrostatic term in electrochemical potential can be multiplied by $(1-C) /(1+C)$ in order to make it applicable in both electrolyte and metal:

$$
\mu=-\alpha \nabla^{2} C+\beta \Psi^{\prime}(C)+4 F \rho_{M} \frac{1-C}{1+C} \Phi .
$$

This electrochemical potential then determines the flux. 


\subsection{Kinetics}

The transport equation in a fluid with velocity field $\vec{u}$ is:

$$
\frac{D C}{D t}=\frac{\partial C}{\partial t}+\vec{\nabla} \cdot(\vec{u} C)=-\vec{\nabla} \cdot \vec{J},
$$

where where $\frac{D}{D t}$ is the substantial derivative which represents the rate of change in the frame of reference moving with velocity $\vec{u}$, and $\vec{J}$ is the flux. Flux in turn is given by the gradient in electrochemical potential times mobility $\kappa$ :

$$
\vec{J}=-\kappa \vec{\nabla} \mu=-\kappa \vec{\nabla}\left(-\alpha \nabla^{2} C+\beta \Psi^{\prime}(C)+4 F \rho_{M} \frac{1-C}{1+C} \Phi\right) .
$$

For iron and ferrous oxide, the molar density is roughly constant, so this term can be taken outside the gradient. The complete conservation equation is given by:

$$
\frac{D C}{D t}=\vec{\nabla} \cdot\left(\kappa \vec{\nabla}\left(-\alpha \nabla^{2} C+\beta \Psi^{\prime}(C)\right)+4 \kappa F \rho_{M} \frac{1-C}{1+C} \vec{\nabla} \Phi\right)
$$

\subsection{Conservation of charge}

The equation for conservation charge is given by:

$$
\frac{D \rho_{f}}{D t}=-\vec{\nabla} \cdot\left(F \rho_{M} \sum z_{i} \vec{J}_{i}-\sigma_{e}(C) \vec{\nabla} \Phi\right),
$$

where $\rho_{f}$ is the charge density and $\sigma_{e}(C)$ is the electronic conductivity. Electronic conductivity in turn is estimated by interpolation between that of the metal and zero in the slag. By comparing the timescales of diffusion and electromigration, of convective charge transport and current, and timescales of diffusion vs. charge buildup at interfaces, in a mass transfer-limited situation with rapid charge redistribution this can be reduced to:

$$
0=\vec{\nabla} \cdot\left(\sigma_{e f f}(C) \vec{\nabla} \Phi\right),
$$

where the effective conductivity $\sigma_{e f f}$ is given by:

$$
\sigma_{e f f}=4 \kappa F^{2} \rho_{M}^{2} \frac{1-C}{1+C}+\sigma_{e}(C)
$$

In the ternary system, transport of charge due to chemical potential-driven diffusion must also be included.

\subsection{Hydrodynamics}

In the EFESR process, the reaction occurs between liquid phases, and it is necessary to include the effect of fluid flow. The Navier-Stokes equations describe 
conservation of mass and momentum in a fluid system. Using $u, v$ and $\omega$ for $x$ and $y$-velocities and vorticity, the velocity-vorticity form is given by:

$$
\begin{aligned}
\nabla^{2} u+\frac{\partial \omega}{\partial y} & =0 \\
\nabla^{2} v-\frac{\partial \omega}{\partial x} & =0 \\
\frac{\partial \omega}{\partial t}+\vec{u} \cdot \nabla \omega & =\nu \nabla^{2} \omega-N \nabla \times(C \nabla \mu),
\end{aligned}
$$

where $\nu$ is the kinematic viscosity and $N$ is a Weber number $\frac{\gamma D}{\rho u^{2} \epsilon}$. The vorticity transport equation includes the curl of the interfacial tension forcing term $(-C \nabla \mu)$ given by Jacqmin [11], which in turn describes driving force due to curvature of a diffuse interface. We assume uniform fluid properties, and neglect gravity.

\subsection{Simulating streamer formation}

The above equations are implemented in the open source RheoPlast tool [12]. Simulations begin with the three-layer metal-oxide-metal condition shown in figure 1. Periodic boundary conditions are applied in the $x$-direction, and symmetry planes used for $C$ in the y direction. Voltage is set to 0 at the top and 1 at the bottom. Physical parameters are: $\rho_{M}=1.31 \times 10^{5} \mathrm{~mol} / \mathrm{m}^{3}, \sigma=12.0 \Omega^{-1}$ $\mathrm{m}^{-1}$ in the slag, $\sigma_{e}=10^{3} \Omega^{-1} \mathrm{~m}^{-1}$ in the metal.

Figures $1 \mathrm{a}$ and $1 \mathrm{~b}$ show simulated cathode shape evolution under unstable and stable conditions respectively. The instability in the former case leads to not only shape change, but also topology change as droplets break free from the streamers.

\subsection{Stability analysis benchmark}

The Peclet number predicts stability of sinusoidal perturbation of wavelength $\lambda$ :

$$
\mathrm{Pe}=\frac{u_{\text {int }} \lambda}{D}=\frac{\sigma_{i} \Delta \Phi \lambda}{z F \rho_{M} L D}=\frac{\Delta \Phi z F \rho_{M} \varepsilon \lambda}{2 \sqrt{18} \cdot 3.1 \gamma L} .
$$

That is, large Peclet number corresponds to fast plating due to large electric field and conductivity, with small diffusivity and interfacial tension, leading to unstable growth. Barkey's linear stability analysis [13] gives the criterion for stable growth in an unsupported electrolyte:

$$
\frac{\Delta \Phi}{L}<\frac{8 \pi^{2} \gamma}{z F \lambda^{2} \rho_{M}} ; \text { i.e. } \mathrm{Pe}<\frac{8 \pi^{2}}{6.2 \sqrt{18}} \frac{\varepsilon}{\lambda}=3.00 \frac{\varepsilon}{\lambda} .
$$

This holds at the limit of a sharp interface, perfect cathode conductivity, infinite electrode separation, and solid electrode i.e. infinite viscosity.

Simulations show that the critical quantity $\mathrm{Pe}_{\text {crit }} \lambda / \epsilon$ approaches 3.0 in the limits of sharp interface, perfect cathode conductivity, infinite electrode separation, 

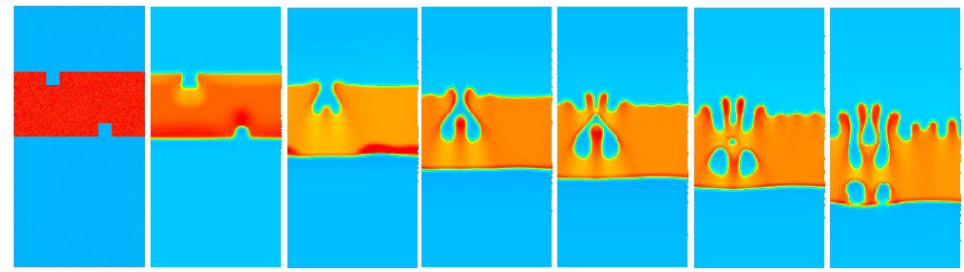

$$
\begin{aligned}
& \Phi=2 * 10^{-8} \\
& \gamma=0.1 \\
& \varepsilon / \lambda=0.039 \\
& \mathrm{~L} / \lambda=0.5 \\
& \mathrm{Pe}=3.95
\end{aligned}
$$

(a) Unstable simulation with high electric field on a $150 \times 300$ grid.
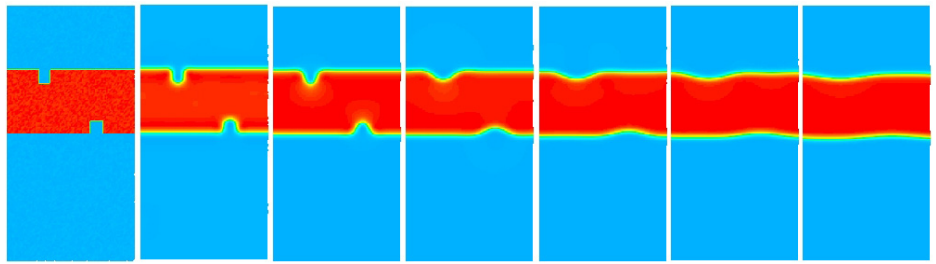

$\Phi=2 * 10^{-8}$

$\gamma=10.0$

$\varepsilon / \lambda=0.039$

$\mathrm{L} / \lambda=0.5$

$\mathrm{Pe}=0.074$

(b) Stable simulation with high interfacial tension on an $80 \times 160$ grid.

Figure 1: Cathode shape evolution without flow under conditions leading to (a) unstable and (b) stable behavior.

and solid electrode or infinite viscosity. That limiting value does not change appreciably as interface thickness approaches $\lambda / 10$, nor for electrode separation as small as $\lambda$.

With liquid electrode and electrolyte, stability is determined by the Schmidt number, which is the ratio $\nu / D$. When the Schmidt number is large, high viscosity prevents flow, and stability behavior approaches the case without flow. When it is small, the interface becomes more stable, as described in further detail in ref [6].

\subsection{Relating the model to experiments}

In the EFESR experiments of $\mathrm{Pal}$ et al., liquid $\mathrm{FeO}$ has the following properties: $\mu=0.20 \mathrm{~Pa} \cdot \mathrm{s}$ [14], $D=4.20 \times 10^{-7} \mathrm{~m}^{2} / \mathrm{s}$ [15], $\rho=3.50 \times 10^{3} \mathrm{~kg} / \mathrm{m}^{3}$, $\gamma=6.45 \times 10^{-1} \mathrm{~N} / \mathrm{m}$ [16]. $S c$ is thus $1.40 \times 10^{2}$, and the critical Peclet number is approximately $2.35 \times 10^{2}$. Experiments produce an electric field of $20 \mathrm{~V} / \mathrm{m}$, resulting in critical wavelength $\lambda_{\text {crit }}=0.4 \mathrm{~mm}$.

One experiment quenched the slag-metal system at the cathode, and the streamers measured approximately $\frac{1}{4} \mathrm{~mm}$ across, corresponding to a critical wavelength approximately twice that. The results of this binary model can thus be considered in good qualitative agreement with experimental observations.

\section{Ternary titanium/magnesium/chlorine model}

The Kroll process, which has dominated titanium production for the past fifty years, is conducted in an iron crucible, resulting in iron contamination of the sponge product near its walls, reducing the process yield. If the crucible were made 
instead of titanium, none of the product would be discarded, however $\mathrm{TiCl}_{4}$ would react with it to form $\mathrm{TiCl}_{3}$ and $\mathrm{TiCl}_{2}$, compromising the integrity of the walls. The subhalide reduction process of Okabe and Takeda [17] starts with magnesium and $\mathrm{TiCl}_{2}$, eliminating the reaction with a titanium crucible. The overall reaction is:

$$
\mathrm{TiCl}_{2}+\mathrm{Mg} \Rightarrow \mathrm{Ti}+\mathrm{MgCl}_{2}
$$

Here phase field simulates sponge structure formation, which involves electronically-mediated reactions (EMR) between $\mathrm{Ti}^{2+}$ and $\mathrm{Mg}$ which are physically separate but connected by electronic conductors. The Ti-Mg-Cl ternary system is most easily represented by two dimensionless concentration parameters $C_{2}$ and $C_{3}: C_{2}$ is zero in $\mathrm{Mg}$ and $\mathrm{MgCl}_{2}$ and one in $\mathrm{Ti}$ and $\mathrm{TiCl}_{2}$, and $C_{3}$ is zero in the chlorides and one in the metals. The mole fractions of each element are then written:

$$
X_{\mathrm{Ti}}=C_{2}\left(\frac{1}{3}+\frac{2}{3} C_{3}\right) X_{\mathrm{Mg}}=\left(1-C_{2}\right)\left(\frac{1}{3}+\frac{2}{3} C_{3}\right) X_{\mathrm{Cl}}=\frac{2}{3}\left(1-C_{3}\right) .
$$

\subsection{Thermodynamics and kinetics}

As before, free energy includes homogeneous, gradient, and electrostatic terms:

$$
F=\int\left(\Psi\left(C_{2}, C_{3}\right)+K_{22}\left|\nabla C_{2}\right|^{2}+K_{33}\left|\nabla C_{3}\right|^{2}+F \Phi \sum z_{i} c_{i}\right) d V,
$$

where again $c_{i}$ is the molar concentration of species $i$, and we omit cross gradient terms. Homogeneous energy is unknown, and is constructed based on its binaries:

$$
\begin{aligned}
\Psi= & C_{2} \ln C_{2}+\left(1-C_{2}\right) \ln \left(1-C_{2}\right)+\Omega_{12} C_{3} C_{2}\left(1-C_{2}\right)+ \\
& C_{3} \ln C_{3}+\left(1-C_{3}\right) \ln \left(1-C_{3}\right)+\Omega_{13} C_{3}\left(1-C_{3}\right)- \\
& \left(C_{2}-0.5\right)\left(C_{3}-0.5\right) .
\end{aligned}
$$

The first three terms form an ideal solution between chlorides $\left(C_{3}=0\right)$, and a regular solution with a miscibility gap between metals $\left(C_{3}=1\right), \Omega_{12}=3.3$. The next three form a regular solution with miscibility gap between metals and chlorides, $\Omega_{13}=2.5$. The last term drives the reaction in equation 19 .

The chemical potential for each concentration parameter $\mu_{i}$ is again the variation of the free energy functional with gradient coefficients $K_{22}=K_{33}=5 \times 10^{-4}$.

$$
\begin{aligned}
& \mu_{2}=-K_{22} \nabla^{2} C_{2}+\frac{\partial \Psi}{\partial C_{2}} \\
& \mu_{3}=-K_{33} \nabla^{2} C_{3}+\frac{\partial \Psi}{\partial C_{3}}+2 F \rho_{M} \frac{1-C_{3}}{1+C_{3}} \Phi .
\end{aligned}
$$

Conservation equations for each parameter can be written as:

$$
\frac{D C_{2}}{D t}=\frac{\partial C_{2}}{\partial t}+\vec{u} \cdot \nabla C_{2}=M_{22} \nabla^{2} \mu_{2}+M_{23} \nabla^{2} \mu_{3}
$$




$$
\frac{D C_{3}}{D t}=M_{32} \nabla^{2} \mu_{2}+M_{33} \nabla^{2}\left(-K_{33} \nabla^{2} C_{3}+\frac{\partial \Psi}{\partial C_{3}}\right)+B \nabla^{2} \Phi,
$$

where $B$ is an electromigration constant set to one, cross mobilities $M_{23}$ and $M_{32}$ are set to zero, and diagonal mobilities $M_{22}$ and $M_{33}$ are both set to $2 \times 10^{-2}$.

\subsection{Conservation of charge}

As mentioned in section 2.3, conservation of charge in the ternary case must include a flux due to chemical potential gradient, and is thus given by:

$$
0=\vec{\nabla} \cdot\left[\tilde{z} M_{33} F \vec{\nabla}\left(-K_{33} \nabla^{2} C_{3}+\frac{\partial \Psi}{\partial C_{3}}\right)\right]+\vec{\nabla} \cdot\left(\sigma_{e f f} \vec{\nabla} \Phi\right),
$$

where $\tilde{z}$ is a constant describing charge transfer due to diffusion and the effective conductivity and electronic conductivity are written such that $\sigma_{e}$ is zero in the chlorides, and that of titanium is half of that of magnesium:

$$
\sigma_{e f f}=\frac{1-C_{3}}{1+C_{3}}+\frac{\sigma_{e}}{\sigma_{i}}, \sigma_{e}=\sigma_{e 0}\left(3 C_{3}^{2}-2 C_{3}^{3}\right)\left(1-\frac{1}{2} C_{2}\right) .
$$

\subsection{Simulations}

Two two-dimensional simulations are presented here. The first, shown in figure 2 , begins with metal phases below, and chloride phases above. As expected, the titanium grows and magnesium shrinks at the Ti-Mg-chlorides triple line, and chlorides interdiffuse but overall become richer in $\mathrm{MgCl}_{2}$. The titanium phase also grows upward into what is originally $\mathrm{TiCl}_{2}$, and the magnesium phase shrinks away from the triple line, indicating electronically-mediated reactions (EMR). Electric potential is low (red) where electrons are generated at the $\mathrm{Mg}-\mathrm{MgCl}_{2}$ interface and high (blue) where they are absorbed at the $\mathrm{Ti}-\mathrm{TiCl}_{2}$ interface. Thus electrons move from low to high potential through metal phases, allowing the reaction to proceed toward completion.

A second simulation of unstable cathode growth without flow is shown in figure 3. This figure shows the formation of a $\mathrm{TiCl}_{2}$-poor layer above the growing titanium which is considerably thinner in front of the "dendrite" tips, resulting in faster diffusion and enhancing the instability due to electric field concentration.

\section{Summary}

The phase field electrochemistry formulation presented here is a potentially powerful tool for simulation of electrochemical reactions. It is able to model such phenomena as roughness formation during plating from a supported or unsupported electrolyte, and electronically-mediated reactions between interfaces in electronic contact. And it shows good quantitative agreement with linear stability theory in the solid case, and develops a new stability theory for reduction 

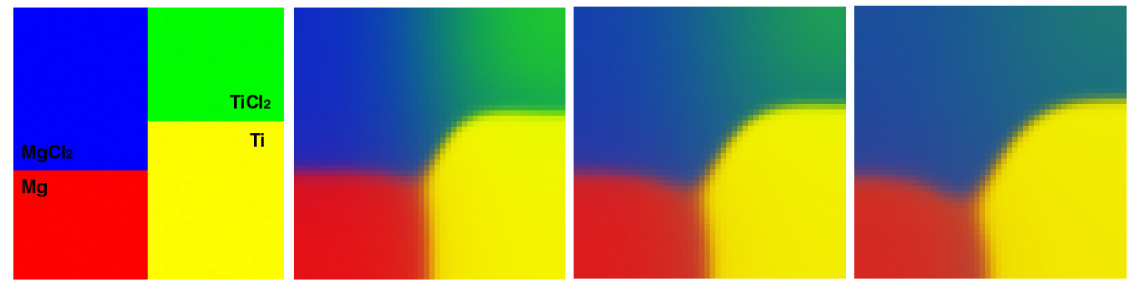

(a) Composition: red=Mg, yellow=Ti, green $=\mathrm{TiCl}_{2}$, blue $=\mathrm{MgCl}_{2}$.
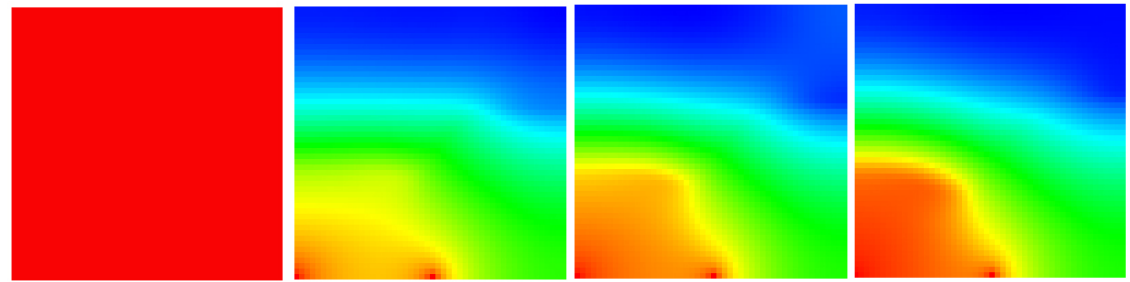

(b) Relative electrical potential: blue at maximum, red at minimum.

Figure 2: Ternary simulation of electronically-mediated reaction (EMR).
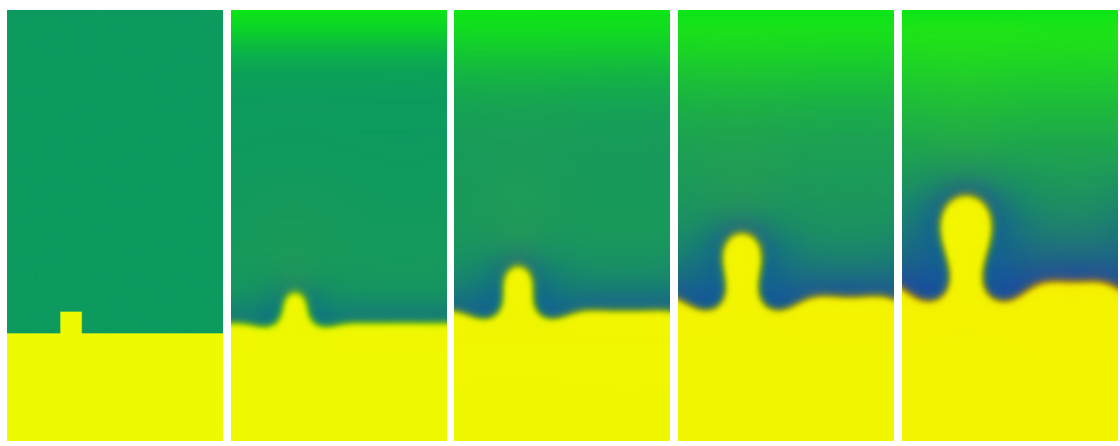

Figure 3: Simulation of titanium deposition, same composition scale as figure 2(a).

of liquid metal from liquid electrolyte which agrees well with an experimental measurement.

The model's assumption of transport-limited systems with negligible charge transfer resistance limits its use, but for high temperature electrometallurgy processes such as the two described here, this model provides a very useful tool.

\section{References}

[1] Cao, Y., Taephaisitphongse, P., Chalupa, R. \& West, A., Linear stability analysis of unsteady galvanostatic electrodeposition in the two-dimensional diffusion-limited regime. J Electrochem Soc, 148, pp. C466-C472, 2001.

[2] Osher, S. \& Sethian, J.A., Fronts propagating with curvature-dependent 
speed: Algorithms based on Hamilton-Jacobi formulations. J Comput Phys, 79, pp. 12-19, 1988.

[3] Wheeler, D., Josell, D. \& Moffat, T., Modeling superconformal electrodeposition using the Level Set Method. J Electrochem Soc, 150, pp. C302-C310, 2003.

[4] Wheeler, D., Josell, D. \& Moffat, T., Influence of catalytic surfactant of roughness evolution during film growth. J Electrochem Soc, 151, pp. C538C544, 2004.

[5] Guyer, J.E., Boettinger, W.J., Warren, J.A. \& McFadden, G.B., Phase field modeling of electrochemistry. II. Kinetics. Phys Rev E, 69(021604), 2004.

[6] Pongsaksawad, W., Powell, A.C. \& Dussault, D., Phase field modeling of transport-limited electrolysis in solid and liquid states. J Echem Soc, (Accepted for publication), 2007.

[7] Pal, U., MacDonald, S., Woolley, D., Manning, C. \& Powell, A., Results demonstrating techniques for enhancing electrochemical reactions involving iron oxide in slags and C in liquid iron. Metall Mater Trans, 36B, pp. 209 218, 2005.

[8] Dussault, D. \& Powell, A., Phase field modeling of electrolysis in a slag or molten salt. Proc. Mills Symp., The Institute of Materials, London, UK, pp. 359-371, 2002.

[9] Cahn, J. \& Hilliard, J. J Chem Phys, 28, p. 258, 1958.

[10] Guyer, J., Boettinger, W., Warren, J. \& McFadden, G., Phase field modeling of electrochemistry. I. Equilibrium. Phys Rev E, 69(021603), 2004.

[11] Jacqmin, D., Calculation of two-phase Navier-Stokes flows using phase-field modeling. J Comp Phys, 155, pp. 96-127, 1999.

[12] Powell, A.C., Zhou, B., Vieyra, J. \& Pongsaksawad, W., RheoPlast phase field multi-physics code. URL http://lyre.mit.edu/ powell/rheoplast.html, currently version 0.8.9.

[13] Barkey, D., Muller, R. \& Tobias, C., Roughness development in metal electrodeposition: II. Stability theory. J Electrochem Soc, 136, pp. 22072214, 1989.

[14] Urbain, G. \& Boiret, M., Ironmaking and steelmaking. National Institute of Standards and Technology, 1990.

[15] Li, Y., Lucas, J., Fruehan, R. \& Belton, G., The chemical diffusivity of oxygen in liquid iron oxide and a calcium ferrite. Metall Mater Trans B, 31B, pp. 1059-1068, 2000.

[16] Mills, K. \& Keene, B., Physical properties of BOS slags. Int Mater Rev, 32, pp. 1-120, 1987.

[17] Okabe, T. \& Takeda, O., A new high speed titanium production by subhalide reduction process. Proc. Light Metals, San Francisco, CA, TMS Annual Meeting, TMS, pp. 1139-1144, 2005. 